\title{
Nature's Chemicals
}

Richard Firn. 2009. Oxford University Press, New York. Pp. 264. \$44.95 (paper). ISBN 9780199603022.

Reviewed by Diana Chen

Reviewer address: 3702 SW Hansom Loop, Bentonville, AR 72712. dkchen@email.uark.edu

Received: February 14, 2013

Volume: 4:30-31

Published: February 20, 2013

C 2013 Society of Ethnobiology

Nature's Chemicals, by plant biologist Richard Firn, is introduced by the author as "a book about ideas." This opening is unexpected because based on the book's subtitle, The Natural Products that Shaped our World, one would think it would be the typical layman's history of economically relevant plant compounds such as coffee, rubber, and quinine a la National Geographic. Instead, as promised, the reader is introduced to ideas concerning not just natural products-the economics, biochemistry, and evolution thereof-but ideas about the nature of science itself. The most famous idea detailed in the book is the "Screening Hypothesis," which Firn developed in 1991 in conjunction with his former graduate student, Clive Jones. As he explains in Chapter 5, the Screening Hypothesis states that to maintain and improve the biodiversity of the world's Natural Products, a certain amount of diversity and flexibility must be present in the biochemical processes that create them. This implies some radical thinking. For example, might some organisms have evolved certain enzymes that are not, as biology students are generally taught, substrate-specific? If this discussion makes the book seem heavy on biochemistry and molecular biology, and perhaps overly didactic, rest assured, it is also an informative and relatively smooth read even for those who don't work in a laboratory. The methodological approach of the book is based in those faceless sciences that Anthropologists commonly eschew. The book outlines the theory behind why The Medicine Man, Sean Connery's Dr. Robert Campbell, would go into the jungle to search for the cure for cancer. That makes it an important read for would-be ethnobotanists, if not as inspiring a one as say, Plotkin's Tales of a Shaman's Apprentice (1994).

In the first chapter, Firn creates a useful convention by defining Natural Products (NPs) as "the distinctive chemicals which characterize particular plant and microbial species (p. 3)." Animals also produce culturally valuable "distinctive chemicals" such as the toxins made by poison dart frogs (Dendrobates spp.) but Firn does not include the study of such chemicals in his book. He does make reference to possible NPs made by humans, but chooses instead to focus on NPs made by plants and microbes that have shaped human history. Firn makes the distinction between NPs (uppercase), which are typically chemicals such as theobromine and caffeine, and nps (lowercase), which are exemplified by consumer products such as cotton and wood. NPs have also been called "Secondary Metabolites" since Albrecht Kossel made the distinction between primary and secondary metabolites in 1891. Firn takes issue with this nomenclature in Chapter 9 of his book, calling it "unhelpful and wrong (p. 9)." Hopefully, for ease of scientific communication, Firn's convention of capitalizing NPs to distinguish them from nps will catch on.

NPs are very important in the daily activities of people around the world, not to mention they are the foundation of major economic systems (both legal and illegal) and yet little is known about them. Most of the world's biochemical diversity - not just biological activity but flavors and scents - is a result of its almost 200,000 different NPs. That may seem like a lot of unique compounds; however, considering the biodiversity of plants and bacteria on Earth, it is apparent that NPs must be present in very few specimens. This book grants the reader a new appreciation for NPs as rare chemicals. Apparently, most NPs come from one of just three main biochemical pathways, and each of those pathways is biologically costly to run, and even more costly to evolve. One of the major points made in the book is that bioprospecting, the in vitro screening for biologically active compounds, is a little-rewarding task. 
Each of the ten chapters begins with a popular quotation and ends with a paragraph or two about "the way science works." These concluding paragraphs are often not reflected in the chapter content and seem like merely a platform from which Firn can vent his frustrations, such as in Chapter 4 a lamentation over the corporate guarding of scientific data. Another one of the beefs Firn has with the way science works, is that it has become too compartmentalized to deal with subjects such as NPs, which require a holistic approach (Chapter 1). The book contains no grand closing chapter to summarize and connect Firn's many interesting ideas. Ethnobotanists would be quite eager to know how the latter chapters that are heaviest in biochemistry and molecular biology, as well as most deficient in the simple black and white illustrations that break up the text in the rest of the book, relate back to its second chapter.

Chapter 2 is the one that will most interest Ethnobotanists. Therein lie the actual narratives of NPs that have shaped our world. The chapter begins with an overview of NPs in Economics and History and then stops to focus on specific NPs such as coffee, cocoa, and opium, and finishes up with NPs in a smattering of other areas such as sodas and perfumes. Although penicillin is included in the book, many other NPs important to the pharmaceutical and health industries such as digitalis and echinacea are absent. It would also have been interesting to see Firn address the recent craze for antioxidants and phytochemicals. In Chapter 4, he does enter the natural vs. synthetic debate, to state predictably that the end products (using the example of vanillin, used in baking) are the same.

Many other books tell the stories of "natural products that shaped our world" in more detail, such as Balick and Cox's Plants, People, and Culture (1996). Specific NPs and their influence on civilization are the subjects of still other books, such as Mintz's Sweetness and Power (1985), a book about sugar. However, what makes Nature's Chemicals a unique book, is how few scientists have taken Firn's holistic approach to explain why NPs evolved, why they are important, and how we can continue to search for and learn about them. Sadly, the free-thinking author died shortly after writing the book. He was 65 years old. 